\title{
Performance of Downlink NOMA in Vehicular Communication Networks: An Analysis Based on Poisson Line Cox Point Process
}

\author{
Yanshi Sun, Student Member, IEEE, Zhiguo Ding, Fellow, IEEE, Xuchu Dai, Keivan Navaie, Senior \\ Member, IEEE, Daniel K. C. So, Senior Member, IEEE
}

\begin{abstract}
In this paper we investigate downlink performance in a non-orthogonal multiple access (NOMA) based vehicular communication network. In contrast to the previous works wherein the vehicles are simply assumed to be located anywhere in the coverage area, we consider practical cases where vehicles are located on a system of roads. We then adopt a system model based on Poisson Line Cox Point (PLC) processes, where the road system is represented through a Poisson Line (PL) Process. Vehicles ordered according to their distances to their corresponding roadside units (RSUs) are then served by using power domain NOMA. Adopting analytical tools from the stochastic geometry, we then analyse the outage performance of the system. Numerical results corroborate our analysis and demonstrate how the system settings influence the considered NOMA based V2X network.
\end{abstract}

Index Terms-Poisson line Cox point (PLC) process, vehicular communications, non-orthogonal multiple access (NOMA)

\section{INTRODUCTION}

$\mathbf{T}$ He proliferation of intelligent transport systems (ITSs) encourages research on design and performance evaluation of vehicle-to-everything (V2X) communications both in academia and industry [1]. According to [2], V2X communications can be implemented either as a sidelink for direct communications over the PC5 interface, such as vehicle-tovehicle (V2V), vehicle-to-pedestrian (V2P) and vehicle-toinfrastructure (V2I), or as a traditional cellular link over the $\mathrm{Uu}$ interface, such as vehicle-to-network (V2N). It is worth pointing out that this paper will only focus on a downlink V2N scenario, where each roadside unit (RSU) needs to transmit different messages to different vehicle users.

Existing V2X communications are based on orthogonal multiple access (OMA), where one channel resource block can only be occupied by one user. However, due to the ever increasing number of vehicular users and the scarce bandwidth available for vehicular communications, a dilemma may occur when the number of users is much larger than the number of available resource blocks. As a result, some users have to wait for a period of time before getting service. In this case, the

Copyright (c) 2015 IEEE. Personal use of this material is permitted. However, permission to use this material for any other purposes must be obtained from the IEEE by sending a request to pubs-permissions@ieee.org

The work of Y. Sun and X. Dai was supported by the National Natural Science Foundation of China under Grant 61971391.

Y. Sun and X. Dai are with the CAS Key Laboratory of Wireless-Optical Communications, University of Science and Technology of China, Hefei, 230026, China. (email: sys@mail.ustc.edu.cn, daixc@ustc.edu.cn).

Z. Ding and D. K. C. So are with the Department of Electrical and Electronic Engineering, the University of Manchester, Manchester M13 9PL, U.K. (email:\{zhiguo.ding, d.so\}@manchester.ac.uk).

$\mathrm{K}$. Navaie is with the School of Computing and Communications, Lancaster University, Lancaster LA1 4WA, U.K. (e-mail: k.navaie@lancaster.ac.uk). requirement for low latency in $\mathrm{V} 2 \mathrm{X}$ communications cannot be guaranteed. To fulfill the drawback caused by OMA, nonorthogonal multiple access (NOMA) techniques have recently been proposed, to enable multiple users to share one single resource block simultaneously [3]-[6]. The key idea of NOMA is to exploit the disparity among users, e.g., different channel conditions or different levels of target data rates. Note that NOMA can be embedded onto the existing OMA based frameworks, such as orthogonal frequency-division multiple access (OFDMA) used in LTE or NR based V2X communications [2], in a manner called hybrid NOMA. Specifically, in hybrid NOMA, users are divided into multiple groups. Orthogonal resource blocks are allocated to different groups as in conventional OMA. While in each group, users' signals are allocated with different levels of power and are superimposed by using the same resource block. Compared to OMA, NOMA can serve more users within the limited bandwidth and achieve higher spectrum efficiency, which makes itself a key enabling technology for the future development of V2X communication systems.

Some prior research endeavor has been put into the application of NOMA to V2X communications [7]-[9]. In [7], the authors proposed efficient centralized and decentralized schemes for NOMA based V2X broadcasting systems. Further, in [8], the authors investigated the application of NOMA to relay-aided broadcasting/multicasting scenarios, where optimal power allocation is studied. In [9], the authors studied the resource allocation problem for NOMA aided V2X network by applying weighted 3-partite interference hypergraph.

In the past two decades, stochastic geometry has been widely used to analyze the performance of wireless networks by modeling locations of wireless nodes as random point processes. Poisson point process (PPP) and Poisson cluster point process (PCP) based models have been applied to study the performance of NOMA in conventional networks [6], [10]. However, these models are not suitable for V2X networks, because they fail to capture the practical geometric layout of the vehicular system, e.g., vehicles are often located on an actual road, instead of uniformly distributed in the plane. On the other hand, Poisson line Cox point (PLC) process [11] has been recognized as an effective model for $\mathrm{V} 2 \mathrm{X}$ communications, where a road system can be modeled as a Poisson line (PL) process and wireless nodes as a 1D point process on each road. The authors in [12] investigated the statistical properties of PLC process for modeling V2X network. The authors then studied the coexistence of base stations uniformly distributed in the plane with base stations located on roads in [13]. 


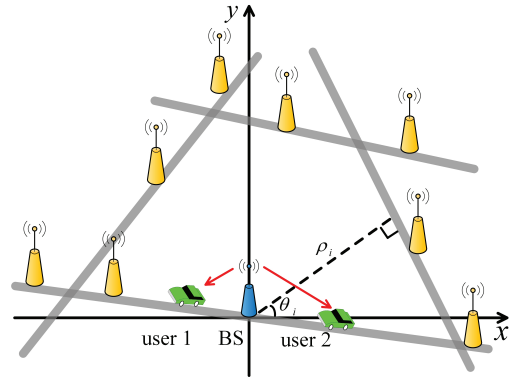

(a)

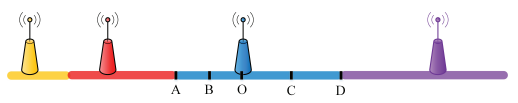

(b) $1 \mathrm{D}$ Voronoi segment, $\delta=2$

Fig. 1: System model.

Further, [14] and [15] studied the performance of a typical user in a vehicular network where transceiver nodes are located on roads modeled by PLC. However, the analytical frameworks developed in these existing works [12]-[15], where one base station serves one user, are not applicable to the modeling and analysis for NOMA. This is because in NOMA, the features (e.g., the locations) of multiple users in a NOMA cluster need to be jointly characterized.

To address the above issues, this paper proposes a realistic model for NOMA in V2X communication systems based on PLC process. In this model, the road system is modelled by using a PL process, where the RSUs are located along the roads based on a 1D PPP. In addition, vehicles served by a given RSU using NOMA technology are also restricted to be along roads and are distributed uniformly within the central part of the corresponding 1D Voronoi segment of the RSU (Defined in section II-A). This is because the vehicles located on the cell edges are better served through coordinated transmission via multiple RSUs.

Adopting this model, we then obtain the intra-line and inter-line interference by applying stochastic geometry theory, based on which we further obtain the outage probability for the NOMA served vehicles. Numerical results corroborate our analysis and demonstrate that a NOMA V2X network outperforms its OMA counterpart.

Notations: $\operatorname{Pr}(\cdot)$ denotes the probability of an event, $\mathbb{E}\{\cdot\}$ denotes the expectation. $\cap$ and $\cup$ denote the intersection and union operation, respectively. $C N(a, b)$ denotes the distribution of a circularly symmetric complex Gaussian random variable with mean $a$ and variance $b$.

\section{SYSTEM MODEL}

To begin with, the definition of PL process and PLC process are given as follows:

Definition 1. A line process is a random collection of lines, which can be denoted by $\Phi_{l}=\left\{l_{i}\right\}$. Note that, each line $l_{i} \in \Phi_{l}$ can be uniquely determined by a point denoted by $\left(\rho_{i}, \theta_{i}\right)$ on a cylindrical surface $\mathcal{C}:=\mathbb{R}^{+} \times[0,2 \pi)$, where $\rho_{i}$ is the perpendicular distance from $l_{i}$ to the origin, and $\theta_{i}$ is the counterclockwise included angle between $x$-axis and the perpendicular, as shown in Fig. 1. Mathematically, the relationship between $l_{i}$ and $\left(\rho_{i}, \theta_{i}\right)$ can be expressed as

$$
l_{i}=\left\{(x, y) \in \mathbb{R}^{2} \mid x \cos \left(\theta_{i}\right)+y \sin \left(\theta_{i}\right)=\rho_{i}\right\} .
$$

Thus, a line process can be generated by a point process $\Xi$ defined on $\mathcal{C}$, by following the one-to-one mapping relationship in (1). Particularly, if $\Xi$ is a PPP on $\mathcal{C}$, then the corresponding generated line process is a PL process [11, Section 8.2.2], [16].

Definition 2. A PLC process $\bar{\Phi}\left(\lambda_{l}, \lambda_{t}\right)$ is a doubly stochastic process which can be generated by the following two steps:

(a). generate a PL process $\Phi_{l}$ by $\Xi$ with intensity $\lambda_{l}$, according to (1);

(b). generate randomly distributed points on each line of $\Phi_{l}$, according to a ID PPP with intensity $\lambda_{t}$, where the collection of points on line $l_{i}$ is denoted by $\Psi_{i}$.

The PLC process $\bar{\Phi}\left(\lambda_{l}, \lambda_{t}\right)$ driven by $\Phi_{l}$ is given by $\bar{\Phi}\left(\lambda_{l}, \lambda_{t}\right):=\left\{\Psi_{i}\right\}_{l_{i} \in \Phi_{l}}$, which means that $\bar{\Phi}\left(\lambda_{l}, \lambda_{t}\right)$ is the collection of points on all lines of $\Phi_{l}$ [14], [16].

Based on the above two definitions, the layout of the road system and the wireless nodes are described in the following subsection.

\section{A. Spatial Modeling of the RSUs and Users}

The RSUs are distributed according to a PLC process $\bar{\Phi}\left(\lambda_{l}, \lambda_{b}\right)$. Specifically, the layout of roads is distributed according to a PL process $\Phi_{l}=\left\{l_{i}\right\}$ generated by a PPP $\Xi$ on $\mathcal{C}$ with intensity $\lambda_{l}$, where $l_{i}$ denotes the $i$-th road. Given the PL process $\Phi_{l}$, the RSUs are distributed according to a 1D PPP denoted by $\Psi_{i}=\left\{x_{m}^{l_{i}}\right\}$ with intensity $\lambda_{b}$ on each line, where $x_{m}^{l_{i}}$ is the $m$-th RSU on $l_{i}$. A RSU is randomly chosen from the PLC process and is termed as the typical RSU. For notational convenience, we set the location of the typical RSU as the origin, which means that we condition on a point at the origin of the PLC process. Further, according to the stationarity of the PLC process [14] and Slivnyak's theorem [17, Section 8.5], conditioning on a point at the origin of the PLC process is equivalent to:

- Firstly, add an additional line $l_{0}$ which passes through the origin to the aforementioned PL process $\Phi_{l}$.

- Secondly, add an additional point (i.e., the typical RSU) at the origin to the 1D PPP on line $l_{0}$.

Hence, under the Palm distribution of the PLC presented above, by conditioning on the typical RSU at the origin, we equivalently obtain a new point process which is given by $\tilde{\Phi}=\bar{\Phi}\left(\lambda_{l}, \lambda_{b}\right) \cup \Psi_{0} \cup\{O\}$, where $\Psi_{0}$ is the 1D PPP on line $l_{0}$. The typical RSU is referred to as the 0 -th RSU on line $l_{0}$.

To yield tractable analysis, this paper only focuses on the performance of users which are served by the RSUs located on the same line. Each line is cut into a series of segments called 1D Voronoi segments, each of which is covered by a RSU. The $m$-th RSU on line $l_{i}$ is closer to the points in its 1D Voronoi segment than other RSUs on line $l_{i}$. The 1D Voronoi segment covered by the $m$-th RSU on line $l_{i}$, which is denoted by $V_{i, m}$, can be mathematically denoted by:

$$
V_{i, m}=\left\{y \in l_{i} \mid \forall x_{n}^{l_{i}} \in \Psi_{i},\left\|y-x_{m}^{l_{i}}\right\| \leq\left\|y-x_{n}^{l_{i}}\right\|\right\} .
$$


As shown in Fig. 1(b), segment $A D$ is the 1D Voronoi segment covered by the RSU located at $O$.

Furthermore, it is noteworthy that this paper focuses on the performance of the users in the center part of each 1D Voronoi segment. The center part of the 1D Voronoi segment covered by the $m$-th RSU on line $l_{i}$, which is denoted by $V_{i, m}^{c}$, is characterized by a parameter $\delta(\delta \geq 1)$ as:

$$
V_{i, m}^{c}=\left\{y^{\prime} \in l_{i} \mid \exists y \in V_{i, m},\left\|y^{\prime}-x_{m}^{l_{i}}\right\|=\frac{1}{\delta}\left\|y-x_{m}^{l_{i}}\right\|\right\} .
$$

As shown in Fig. 1(b), when $\delta=2$, the center part of the 1D Voronoi segment located at $O$ is segment $B C$, where $O B=$ $\frac{1}{\delta} O A$ and $O C=\frac{1}{\delta} O D$.

It is assumed that the user density is dense enough so that there are at least $K$ users in the center part of each 1D Voronoi segment. Then, $K$ users are chosen from this center part and are grouped together to be served by NOMA by using the same channel resource block. The selected $K$ users are uniformly distributed in the center part of each 1D Voronoi segment independently and identically. It is noteworthy that these selected users of all the 1D Voronoi segments in the whole network form a Type I user point process [18], whose performance is the focus of this paper.

Remark 1. Note that, in (3), for $\delta=1, V_{i, m}^{c}$ is exactly $V_{i, m}$. While for $\delta>1$, users located at the edge part of the cell are excluded from our analysis. Because these edge users are relatively far from their RSUs in some scenarios, solely applying NOMA to such users will result in a poor performance. In practice, such users might be served by using coordinated transmission through multiple RSUs.

\section{B. NOMA Transmission}

Users are ordered according to their distances to the typical RSU, i.e. $D_{0, i} \leq D_{0, j}, 1 \leq i \leq j \leq K$, where $D_{0, k}$ is the distance of the $k$-th user from the typical RSU. The transmitted signal at the typical RSU is therefore:

$$
s_{0}^{l_{0}}=\sum_{k=1}^{K} \sqrt{\beta_{k} P} s_{0, k}^{l_{0}},
$$

where $s_{0, k}^{l_{0}}$ is the message intended for user $k$ with normalized power, $P$ is the total power of a RSU, and $\beta_{k}$ is the power ratio allocated to user $k$, where $\sum_{k=1}^{K} \beta_{k}=1$. In addition, according to NOMA principle, far users are allocated with more powers, which means the power allocation coefficients should satisfy the following constraint:

$$
\beta_{1}<\beta_{2}<\cdots<\beta_{K}
$$

At the receiver side, the observed signal by user $k$ is:

$$
y_{k}=\tilde{h}_{0, k} s_{0}^{l_{0}}+\sum_{x_{m}^{l_{0} \in \Psi_{0}}} \tilde{h}_{m, k} s_{m}^{l_{0}}+\sum_{l_{i} \in \Phi_{l}} \sum_{x_{m}^{l_{i}} \in \Psi_{i}} \hat{h}_{i, m, k} s_{m}^{l_{i}}+n_{k},
$$

where $\tilde{h}_{m, k}=\frac{\tilde{g}_{m, k}}{\tilde{D}_{m, k}^{\alpha_{0} / 2}}$ is the the channel gain between the RSU located at $x_{m}^{l_{0}}$ and user $k, \tilde{g}_{m, k}$ is the Rayleigh distributed small scale fading, i.e., $\tilde{g}_{m, k} \sim C N(0,1), \tilde{D}_{m, k}$ is the distance between user $k$ and $x_{m}^{l_{0}}$, and $\alpha_{0}$ is the corresponding large scale path loss exponent. Note that $m=0$ denotes the typical RSU. Similarly, $\hat{h}_{i, m, k}=\frac{\hat{g}_{i, m, k}}{\hat{D}_{i, m, k}^{\alpha / 2}}$ is the channel gain between the $m$-th RSU located on $l_{i}$ and user $k, \hat{g}_{i, m, k}$ is the Rayleigh small scale fading, i.e., $\hat{g}_{i, m, k} \sim C N(0,1), \hat{D}_{i, m, k}$ is the distance between user $k$ and $x_{m}^{l_{i}}$, and $\alpha_{1}$ is the large scale path loss exponent. The additive noise is also represented by $n_{k}$ as a circular symmetric complex Gaussian random variable, i.e., $n_{k} \sim C N\left(0, \sigma^{2}\right)$, with $\sigma^{2}$ being the noise power. It is noteworthy that, by considering the electromagnetic propagation environment between two nodes on the same road and on different roads are different, we use different notations for the path loss exponents, i.e., $\alpha_{0}$ and $\alpha_{1}$.

Remark 2. Block fading is assumed in this paper, because the user mobility can be neglected in a given time slot and the channel can be treated as unchanged. A promising direction for future work is that the effect caused by user mobility can be studied. Due to its mobility, a vehicular user may be handed over from one RSU to another. Besides, the distance between the moving user and the corresponding associated RSU may also changes, which means that the user's order in a NOMA cluster needs to be continually adjusted. Thus, it will be interesting to investigate a moving user's handover rate and average outage probability over a long term.

According to NOMA principle, user $k$ needs to carry out SIC to remove the signals intended for all users which have weaker channel conditions than user $k$, i.e., from user $k+1$ to user $K$. The signal-to-interference-plus-noise ratio (SINR) at user $k$ for decoding the signal intended for user $n(k \leq n \leq$ $K)$ is given by

$$
\operatorname{SINR}_{k}^{n}=\frac{\left|\tilde{h}_{0, k}\right|^{2} \beta_{n}}{\sum_{j=1}^{n-1} \beta_{j}\left|\tilde{h}_{0, k}\right|^{2}+I_{k}+1 / \rho},
$$

where $\rho=P / \sigma^{2}$ is the transmit signal-to-noise ratio (SNR), $I_{k}$ is the interference received from other RSUs:

$$
I_{k}=\underbrace{\sum_{x_{m}^{l_{0} \in \Psi_{0}}}\left|\tilde{h}_{m, k}\right|^{2}}_{I_{k}^{\text {intra }}}+\underbrace{\sum_{x_{i} \in \Phi_{l}} \sum_{x_{x_{i}}^{l_{i} \in \Psi_{i}}}\left|\hat{h}_{i, m, k}\right|^{2}}_{I_{k}^{\text {inter }}} .
$$

Note that in (8), $I_{k}^{\text {intra }}$ is the interference from the same road, termed "intra-line interference", and $I_{k}^{\text {inter }}$ is the interference from other lines, termed "inter-line interference".

The outage probability of user $k$ is therefore:

$$
P_{k}^{\text {out }}=1-\operatorname{Pr}\left(\bigcap_{n=k}^{K} \operatorname{SINR}_{k}^{n}>\epsilon_{n}\right)
$$

where $\epsilon_{n}=2^{\bar{R}_{n}}-1, \bar{R}_{n}$ is user $n$ 's target data rate with unit bit per channel use (BPCU).

\section{PERformance EVAluation}

This paper adopts outage probability as the metric to evaluate the performance achieved by the considered NOMA aided vehicular communication network. The reasons for using outage probability as the metric are two folds. Firstly, the outage probability gives a tight bound for the error probability 
of detection. Secondly, the outage probability can be adopted to calculate the capacity.

To obtain the outage probabilities achieved by the users, we first characterize the following distance distributions and Laplace transform of the interferences.

On line $l_{0}$, there are two neighboring RSUs of the typical RSU, the one which is closer to the typical RSU than the other denoted by $x_{1}^{l_{0}}$, and the other denoted by $x_{2}^{l_{0}}$. Denote $R_{1}=\left\|x_{1}^{l_{0}}\right\|$ and $R_{2}=\left\|x_{2}^{l_{0}}\right\|$ as the distances from the typical RSU to its adjacent RSUs, where we have $R_{1} \leq R_{2}$. The joint probability density function (pdf) of the ordered $R_{1}$ and $R_{2}$ is:

$$
f_{R_{1}, R_{2}}\left(r_{1}, r_{2}\right)=2 \lambda_{b}^{2} e^{-\lambda_{b}\left(r_{1}+r_{2}\right)}, 0<r_{1} \leq r_{2} .
$$

Since the $K$ users are uniformly distributed within the center part of the 1D Voronoi segment covered by the typical RSU, i.e., $V_{0,0}^{c}$, given $R_{1}=r_{1}$ and $R_{2}=r_{2}$, the pdf of the distance between an arbitrary unordered user and the typical RSU, $D_{0}$, is:

$$
f_{D_{0}}\left(d \mid r_{1}, r_{2}\right)= \begin{cases}\frac{4 \delta}{r_{1}+r_{2}}, & 0<d \leq \frac{r_{1}}{2 \delta} \\ \frac{2 \delta}{r_{1}+r_{2}}, & \frac{r_{1}}{2 \delta}<d \leq \frac{r_{2}}{2 \delta}\end{cases}
$$

Utilising the notion of order statistics [19], the corresponding pdf of the distance between the $k$-th ordered user and the typical RSU is the following:

$f_{D_{0, k}}\left(d \mid r_{1}, r_{2}\right)=\left\{\begin{array}{cl}\frac{(4 \delta)^{k} K ! d^{k-1}\left(r_{1}+r_{2}-4 \delta d\right)^{K-k}}{(k-1) !(K-k) !\left(r_{1}+r_{2}\right)^{K}}, & 0<d \leq \frac{r_{1}}{2 \delta}, \\ \frac{2 \delta K !\left(r_{1}+2 \delta d\right)^{k-1}\left(r_{2}-2 \delta d\right)^{K-k}}{(k-1) !(K-k) !\left(r_{1}+r_{2}\right)^{K}}, & \frac{r_{1}}{2 \delta}<d \leq \frac{r_{2}}{2 \delta}\end{array}\right.$

For a given user $k$, Laplace transform of the intra-line interference $I_{k}^{\text {intra }}$ and the inter-line interference $I_{k}^{\text {inter }}$ are characterized in the following two lemmas.

Lemma 1. For $R_{1}=r_{1}, R_{2}=r_{2}, D_{0, k}=d$, Laplace transform of the interference from the RSUs on $l_{0}$, i.e., $\mathcal{L}_{k}^{\text {intra }}\left(s \mid r_{1}, r_{2}, d\right) \triangleq \mathbb{E}\left\{e^{-s I_{k}^{\text {intra }}}\right\}$, is given in the following:

$\mathcal{L}_{k}^{\text {intra }}\left(s \mid r_{1}, r_{2}, d\right)= \begin{cases}\frac{1}{2} F\left(s, r_{1}-d, r_{2}+d\right)+ & \\ \frac{1}{2} F\left(s, r_{1}+d, r_{2}-d\right), & 0<d \leq \frac{r_{1}}{2 \delta}, \\ F\left(s, r_{1}+d, r_{2}-d\right), & \frac{r_{1}}{2 \delta}<d \leq \frac{r_{2}}{2 \delta} .\end{cases}$

where

$$
\begin{aligned}
F(s, u, v)= & \frac{\exp \left(-\lambda_{b} \frac{s u^{1-\alpha_{0}}}{\alpha_{0}-1}{ }_{2} F_{1}\left(1,1-\frac{1}{\alpha_{0}} ; 2-\frac{1}{\alpha_{0}} ;-\frac{s}{u^{\alpha_{0}}}\right)\right)}{\left(1+s / u^{\alpha_{0}}\right)} \\
& \cdot \frac{\exp \left(-\lambda_{b} \frac{s v^{1-\alpha_{0}}}{\alpha_{0}-1}{ }_{2} F_{1}\left(1,1-\frac{1}{\alpha_{0}} ; 2-\frac{1}{\alpha_{0}} ;-\frac{s}{v^{\alpha_{0}}}\right)\right)}{\left(1+s / v^{\alpha_{0}}\right)},
\end{aligned}
$$

where ${ }_{2} F_{1}(\cdot)$ is the Gauss hyper-geometric function [20].

Proof: Please refer to Appendix A.

Lemma 2. Laplace transform of the received interference from RSUs located on the other lines, i.e., $\mathcal{L}^{\text {inter }}(s) \triangleq$ $\mathbb{E}\left\{e^{-s I_{k}^{\text {inter }}}\right\}$, is:

$$
\mathcal{L}_{k}^{\text {inter }}(s)=\exp \left(-2 \pi \lambda_{l} \int_{0}^{\infty}(1-G(x, s)) d x\right),
$$

where

$$
G(x, s)=\exp \left(-2 \lambda_{b} \int_{0}^{\infty} \frac{s}{s+\left(x^{2}+u^{2}\right)^{\frac{\alpha_{1}}{2}}} d u\right) .
$$

It is noteworthy that the results in Lemma 2 have been provided in the literature [15], [21]. To make the paper selfcontained, a brief proof for Lemma 2 is provided in Appendix B.

Remark 3. The Laplace transform of the inter-line interference modeled by PLC is different from and more complex than that modeled by PPP. For example, as shown in Appendix $B$, when evaluating the Laplace transform of the inter-line interference modeled by $P L C$, we need to apply the probability generating functional (PGFL) twice, since PLC is a doubly stochastic process. While for PPP, we only need to apply PGFL once.

Using the results of Lemmas 1 and 2, the outage probability for a user $k(1 \leq k \leq K)$ which is served by the typical RSU is given in Theorem 1 .

Theorem 1. The outage probability for user $k, 1 \leq k \leq K$, in the typical cell is:

$$
P_{k}^{\text {out }}=1-\int_{0}^{\infty} \int_{0}^{r_{2}} P_{k}\left(r_{1}, r_{2}\right) f_{R_{1}, R_{2}}\left(r_{1}, r_{2}\right) d r_{1} d r_{2},
$$

where $P_{k}\left(r_{1}, r_{2}\right)$ is the conditional coverage probability for $R_{1}=r_{1}$ and $R_{2}=r_{2}$ as the following:

$$
\begin{gathered}
P_{k}\left(r_{1}, r_{2}\right)=\int_{0}^{\frac{r_{2}}{2}} \mathcal{L}^{\text {intra }}\left(y^{\alpha_{0}} \tilde{\epsilon}_{k} \mid r_{1}, r_{2}, y\right) \mathcal{L}^{\text {inter }}\left(y^{\alpha_{0}} \tilde{\epsilon}_{k}\right) \\
e^{-\tilde{\epsilon}_{k} y^{\alpha_{0}} / \rho} f_{D_{0, k}}\left(y \mid r_{1}, r_{2}\right) d y,
\end{gathered}
$$

where $\tilde{\epsilon}_{k}=\max _{k \leq n \leq K}\left\{\frac{\epsilon_{n}}{\beta_{n}-\epsilon_{n} \sum_{j=1}^{n-1} \beta_{j}}\right\}$.

Proof: The outage probability $P_{k}^{\text {out }}$ shown in (9) can be rewritten as

$$
P_{k}^{\text {out }}=1-\operatorname{Pr}\left(\left|\tilde{h}_{0, k}\right|^{2}>\tilde{\epsilon}_{k}\left(I_{k}+1 / \rho\right)\right),
$$

which is

$$
P_{k}^{\text {out }}=1-\mathbb{E}_{r_{1}, r_{2}}\left\{\mathbb{E}_{d}\left\{\mathbb{E}_{I_{k}}\left\{e^{-\tilde{\epsilon}_{k} d^{\alpha_{0}}\left(I_{k}+1 / \rho\right)}\right\}\right\}\right\},
$$

that completes the proof.

Corollary 1. When $\lambda_{l} \rightarrow \infty$ and $\lambda_{l} \lambda_{b}=\lambda$, where $\lambda$ is a constant, the conditional coverage probability shown in (18) can be approximated as:

$$
P_{k}\left(r_{1}, r_{2}\right) \approx \int_{0}^{\frac{r_{2}}{2}} Q\left(y^{\alpha_{0}} \tilde{\epsilon}_{k}\right) e^{-\tilde{\epsilon}_{k} y^{\alpha_{0}} / \rho} f_{D_{0, k}}\left(y \mid r_{1}, r_{2}\right) d y
$$

where

$$
Q(s)=\exp \left(-\frac{2 \pi^{2} \lambda s^{\frac{2}{\alpha_{1}}}}{\alpha_{1}} B\left(\frac{2}{\alpha_{1}}, 1-\frac{2}{\alpha_{1}}\right)\right) .
$$

Remark 4. Note that in (21), the intra-line interference term disappears, since $\lambda_{b}$ tends to zero. Besides, $Q(s)$ is the same as the Laplace transform of a $2 D$ PPP with intensity $\pi \lambda_{l} \lambda_{b}$, which implies that the inter-line interference degrades to a $2 D$ PPP under the considered limit case. 


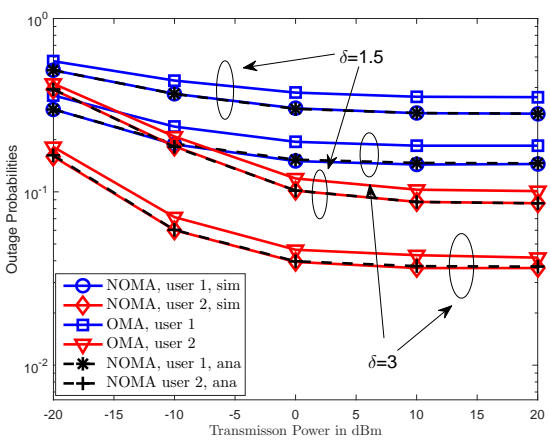

Fig. 2: Outage probabilities versus transmit power for NOMA and OMA schemes, where $\lambda_{l}=2 \times 10^{-3}, \lambda_{b}=10^{-2}$.

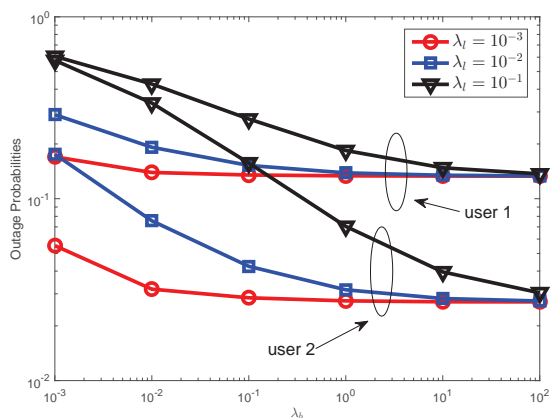

Fig. 3: Outage probabilities versus $\lambda_{b}$ for various $\lambda_{l}$ values, where $P=30 \mathrm{dBm}, \delta=3$.

\section{NUMERICAL RESULTS}

In this section, numerical results are provided to demonstrate the achieved performance by NOMA in the considered PLC process modeled vehicular network, and also to validate the correctness of the analysis. Unless stated otherwise, the parameters are set as follows. The number of users is set as $K=2$. The background noise power is $-170 \mathrm{dBm} / \mathrm{Hz}$, the carrier frequency is set as $2 \mathrm{GHz}$, the occupied bandwidth of the transmission is $10 \mathrm{MHz}$. The path loss exponents are set as $\alpha_{0}=3$ and $\alpha_{1}=4$. The target data rates of the two users are set as $\bar{R}_{1}=2 \mathrm{BPCU}$ and $\bar{R}_{2}=0.2 \mathrm{BPCU}$. The power allocation coefficient is set as $\beta_{1}=\frac{4}{11}$. The simulation results are obtained by using Monte Carlo simulations via MATLAB. Specifically, to obtain each point in the simulation results, we do 100000 independent realizations of the PLC process, and then average over these realizations.

Fig. 2 shows the outage probabilities achieved by users served by NOMA and OMA. The benchmark OMA scheme in Fig. 2 is based on time-division multiple access (TDMA), where $\frac{1}{K}$ of the time slot is allocated to each of the $K$ users and the transmit power is $P$. It is seen in Fig. 2 simulation results perfectly match the analytical results, which verifies the accuracy of our analysis. It is also seen that, NOMA provides a lower outage probability for both users compared to OMA. This indicates that by a proper design, NOMA can outperform OMA.

Fig. 3 shows the impact of $\lambda_{l}$ and $\lambda_{b}$ on the outage performance. From Fig. 3, it can be seen that, for a small $\lambda_{b}$, the outage probabilities increase by increasing $\lambda_{l}$. This is because the inter-line interference level is increased by increasing $\lambda_{l}$, which in return degrades the outage performance. However,

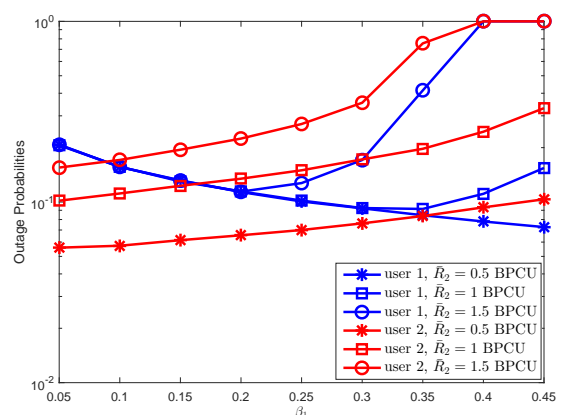

Fig. 4: Impact of power allocation coefficients. $P=20 \mathrm{dBm}$, $\delta=3, \bar{R}_{1}=1$ BPCU.

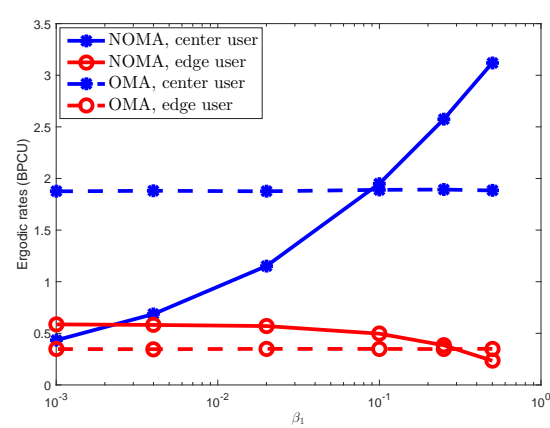

Fig. 5: Ergodic rates achieved by center and edge users. $P=$ $20 \mathrm{dBm}, \delta=1$.

as $\lambda_{b}$ increases, the gap between the plots with different choices of $\lambda_{l}$ becomes narrower. This suggests that for large enough $\lambda_{b}$, the impact of $\lambda_{b}$ on the outage probability is more significant than that of $\lambda_{l}$. From Fig. 3 , it is shown that the outage probability first decreases with $\lambda_{b}$. However, the rate of decrease becomes slower by increasing $\lambda_{b}$. For a very large $\lambda_{b}$, the outage probability converges to a fixed value.

Fig. 4 shows the outage probabilities versus the power allocation coefficient $\beta_{1}$. Since user 2 treats user 1's signal as noise, the outage probabilities achieved by user 2 increase with $\beta_{1}$ as shown in Fig. 4. However, before decoding its own signal, user 1 should first carry out SIC to cancel user 2 's signal. Thus, as shown in Fig. 4, when $\beta_{1}$ is small, the outage probabilities achieved by user 1 of all the three cases decrease with $\beta_{1}$, which means decoding user 1's own signal is the mean limitation. In addition, as $\beta_{1}$ increases, the main limitation for the cases where $\bar{R}_{2}=1 \mathrm{BPCU}$ and $\bar{R}_{2}=1.5$ BPCU turns into removing user 2 's signal, thus the outage probabilities of user 1 turn to increase as $\beta_{1}$ increases.

In Fig. 5, user 2 is set as the worst case edge user, where $D_{0,2}=\frac{r_{2}}{2}$, and user 1 is set as a center user which is randomly distributed in the 1D Voronoi segment. It is shown that when OMA is applied, the data rate achieved by the edge user is much lower than that of the center user. However, in NOMA, with more allocated power, the edge user can achieve a higher rate at the cost of degrading the center user's rate. Hence, NOMA can achieve better fairness for the edge user. However, even allocated with more power in NOMA, the edge user's achievable rate is still very low. Thus, it is better to serve the edge user via coordinated transmission as stated in Remark 1. 


\section{Conclusions}

In this paper, the outage performance achieved by NOMA in vehicular communication networks has been studied. A stochastic geometric model based on PLC process has been applied to represent the spatial topology of a vehicular network. It has been shown that with careful design of the system, NOMA outperforms OMA in vehicular communication networks in terms of outage probability.

\section{APPENDIX A}

\section{PROOF FOR LEMMA 1}

Let $\bar{\Psi}_{0}^{t}(t=1,2)$ be the RSUs which are located on the same side as $x_{t}^{l_{0}}$ with respect to $O$. Denote

$$
\tilde{I}_{t}=\left|\tilde{h}_{t, k}\right|^{2}+\sum_{x_{m}^{l_{0}} \in \bar{\Psi}_{0}^{t}}\left|\tilde{h}_{m, k}\right|^{2},
$$

then we have $I_{\text {intra }}=\tilde{I}_{1}+\tilde{I}_{2}$ and $\mathcal{L}^{\text {intra }}\left(s \mid r_{1}, r_{2}, d\right)=$ $\mathcal{L}^{\tilde{I}_{1}}\left(s \mid \tilde{D}_{1, k}\right) \mathcal{L}^{\tilde{I}_{2}}\left(s \mid \tilde{D}_{2, k}\right)$, where $\mathcal{L}^{\tilde{I}_{t}}\left(s \mid \tilde{D}_{t, k}\right)$ is Laplace transform of $\tilde{I}_{t}$ given $\tilde{D}_{t, k}$ :

$$
\begin{aligned}
\mathcal{L}^{\tilde{I}_{t}}\left(s \mid \tilde{D}_{t, k}\right) & =\frac{1}{1+s / \tilde{D}_{t, k}^{\alpha_{0}}} \mathbb{E}\left\{\prod_{x_{m}^{l_{0} \in \bar{\Psi}_{0}^{t}}} \frac{1}{1+s / \tilde{D}_{m, k}^{\alpha_{0}}}\right\} \\
& =\frac{1}{1+s / \tilde{D}_{t, k}^{\alpha_{0}}} \exp \left(-\lambda_{b} \int_{\tilde{D}_{t, k}}^{\infty} \frac{s}{s+r^{\alpha_{0}}} d r\right) .
\end{aligned}
$$

In (24), the first step follows by taking the expectations with respect to the i.i.d small scale fadings and the second step is obtained by using the PGFL of the 1D PPP [17]. The proof is completed by simply noting the relationship between $\tilde{D}_{t, k}$ and different cases of $d$.

\section{APPENDIX B \\ PROOF FOR LEMMA 2}

Due to the stationarity of the PLC process [14], for calculating Laplace transform of $I_{k}^{\text {inter }}$, it is reasonable to set the location of user $k$ at the origin, i.e., $U_{k}=O$. Then, the Laplace transform of $I_{k}^{\text {inter }}$ can be calculated as in the following:

$$
\begin{aligned}
& \mathcal{L}_{k}^{\text {inter }}(s) \\
& =\mathbb{E}\left\{\exp \left(-s \sum_{l_{i} \in \Phi_{l}} \sum_{x_{m}^{l_{i} \in \Psi_{i}}} \frac{\left|\hat{g}_{i, m, k}\right|^{2}}{\|\left. x_{m}^{l_{i}}\right|^{\alpha_{1}}}\right)\right\} \\
& \stackrel{(a)}{=} \mathbb{E}\left\{\prod_{l_{i} \in \Phi_{l}} \prod_{x_{m}^{l_{i}} \in \Psi_{i}} \frac{1}{1+\frac{s}{\left\|x_{m}^{l_{i}}\right\|^{\alpha_{1}}}}\right\} \\
& \stackrel{(b)}{=} \mathbb{E}\left\{\prod _ { l _ { i } \in \Phi _ { l } } \mathbb { E } \left\{\prod_{x_{m}^{l_{i} \in \Psi_{i}}} \frac{\left.\left.1+\frac{s}{\left(\rho_{i}^{2}+u_{i, m}^{2}\right)^{\alpha_{1} / 2}} \mid l_{i}\right\}\right\}}{\stackrel{(c)}{=}} \mathbb{E}\left\{\prod_{l_{i} \in \Phi_{l}} \exp \left(-2 \lambda_{b} \int_{0}^{\infty} \frac{s}{s+\left(\rho_{i}^{2}+u^{2}\right)^{\alpha_{1} / 2}} d u\right)\right\}\right.\right. \\
& \stackrel{(d)}{=} \exp \left(-2 \pi \lambda_{l} \int_{0}^{\infty} 1-\exp \left(-2 \lambda_{b} \int_{0}^{\infty} \frac{s}{s+\left(x^{2}+u^{2}\right)^{\alpha_{1} / 2}} d u\right) d x\right)
\end{aligned}
$$

where step (a) is obtained by noting that, $\hat{g}_{i, m, k}$ is the Rayleigh small scale fading, which yields $\left|\hat{g}_{i, m, k}\right|^{2}$ is exponentially distributed with parameter 1 . Step (b) is obtained by conditioning on the location of $l_{i}$ in the inner expectation, where $\rho_{i}$ is the distance between the origin and $l_{i}$, and $u_{i, m}$ is the distance between the projection of the origin onto line $l_{i}$ and $x_{m}^{l_{i}}$. Step (c) is obtained by utilising the PGFL of the 1D PPP on line $l_{i}$, and step (d) is obtained by utilising the PGFL of $\Xi$, which is a 2D PPP on the representation space $\mathcal{C}$, [15], [21].

\section{REFERENCES}

[1] S. Chen, J. Hu, Y. Shi, and L. Zhao, "LTE-V: A TD-LTE-based V2X solution for future vehicular network," IEEE Internet Things J., vol. 3 , no. 6, pp. 997-1005, 2016.

[2] 3GPP TR 38.885 V16.0.0, "Study on NR Vehicle-to-Everything (V2X)," Mar. 2019.

[3] Z. Ding, Y. Liu, J. Choi, Q. Sun, M. Elkashlan, I. Chih-Lin, and H. V. Poor, "Application of non-orthogonal multiple access in LTE and 5G networks," IEEE Commun. Mag., vol. 55, no. 2, pp. 185-191, Feb. 2017.

[4] Z. Ding, Z. Yang, P. Fan, and H. V. Poor, "On the performance of non-orthogonal multiple access in 5G systems with randomly deployed users," IEEE Signal Process. Lett., vol. 21, no. 12, pp. 1501-1505, Dec. 2014.

[5] M. Aldababsa, M. Toka, S. Gökçeli, G. K. Kurt, and O. Kucur, "A tutorial on nonorthogonal multiple access for $5 \mathrm{G}$ and beyond," Wireless Commun. Mobile Comput., vol. 2018, Feb. 2018.

[6] Y. Sun, Z. Ding, and X. Dai, "On the performance of downlink NOMA in multi-cell mmWave networks," IEEE Commun. Lett., vol. 22, no. 11, pp. 2366-2369, Nov. 2018.

[7] B. Di, L. Song, Y. Li, and G. Y. Li, "NOMA-based low-latency and high-reliable broadcast communications for 5G V2X services," in Proc. IEEE GLOBECOM, Singapore, Dec. 2017, pp. 1-6.

[8] G. Liu, Z. Wang, J. Hu, Z. Ding, and P. Fan, "Cooperative NOMA Broadcasting/Multicasting for Low-Latency and High-Reliability 5G Cellular V2X Communications," IEEE Internet Things J., vol. 6, no. 5, pp. 7828-7838, Oct. 2019.

[9] B. Wang, R. Zhang, C. Chen, X. Cheng, L. Yang, and Y. Jin, "Interference Hypergraph-Based 3D Matching Resource Allocation Protocol for NOMA-V2X Networks," IEEE Access, vol. 7, pp. $90789-90$ 800, Jul 2019.

[10] Z. Zhang, H. Sun, and R. Q. Hu, "Downlink and uplink non-orthogonal multiple access in a dense wireless network," IEEE J. Select. Areas Commun., vol. 35, no. 12, pp. 2771-2784, Jul. 2017.

[11] S. N. Chiu, D. Stoyan, W. S. Kendall, and J. Mecke, Stochastic geometry and its applications. John Wiley \& Sons, 2013.

[12] C.-S. Choi and F. Baccelli, "Poisson cox point processes for vehicular networks," IEEE Trans. Veh. Technol., vol. 67, no. 10, pp. 10 160-10 165, Jul. 2018.

[13] — , "An analytical framework for coverage in cellular networks leveraging vehicles," IEEE Trans. Commun., vol. 66, no. 10, pp. 49504964, May 2018.

[14] V. V. Chetlur and H. S. Dhillon, "Coverage analysis of a vehicular network modeled as Cox process driven by Poisson line process," IEEE Trans. Wireless Commun., vol. 17, no. 7, pp. 4401-4416, Apr. 2018.

[15] — , "Success probability and area spectral efficiency of a vanet modeled as a cox process," IEEE Wireless Commun. Lett., vol. 7, no. 5, pp. 856-859, 2018.

[16] Y. Sun, Z. Ding, and X. Dai, "On the Outage Performance of Network NOMA (N-NOMA) Modeled by Poisson Line Cox Point Process," 2019, available online at arXiv: 1911.042955.

[17] M. Haenggi, Stochastic geometry for wireless networks. Cambridge, U.K.: Cambridge Univ. Press, 2012.

[18] — "User point processes in cellular networks," IEEE Wireless Commun. Lett., vol. 6, no. 2, pp. 258-261, Feb. 2017.

[19] H. David and H. Nagaraja, Order statistics. 3rd, 3rd ed. Wiley, New York, NY, 2003

[20] I. M.Abramowitz, Handbook of Mathematical Functions. Dover, 1970.

[21] B. Błaszczyszyn and P. Mühlethaler, "Random linear multihop relaying in a general field of interferers using spatial aloha," IEEE Trans. Wireless Commun., vol. 14, no. 7, pp. 3700-3714, Mar. 2015. 